\section{Habits of the Cuckoo.}

ONE day last week I was in my garden-a not particularly private country one-when I heard a cuckoo close by, and, standing quite still, I saw the bird alight upon an apple tree not more than four yards from me. The bird did not appear to object to my close proximity, for it uttered its call " cuckoo" twice. Its mate then came and sat in a plum tree only five yards from me, on the opposite side of me; one of them had a caterpillar in its mouth. Then a blackbird came into another tree in a state of great excitement uttering its " pink pink," as I supposed, at the cuckoos ; and the question arose in my mind, "Does the cuckoo feed its own young, and was that in the blackbird's nest?" Can any of your readers help me? WM. H. WILSON.

Gloucester House, Sudbury, Harrow, June I9.

\section{Economic Entomology.}

CAN you tell me where I can get information as to the present condition of economic entomology in this country, more especially as to methods of research usually adopted?

\section{MAGNETIC PERTURBATIONS OF THE SPECTRAL LINES.}

$T H E$ subject which we are about to consider this evening forms a connecting link between two of the most interesting branches of human knowledge-namely, that which treats of magnetism and that which treats of light. Almost as soon as the properties of magnets became known, mere curiosity alone must have prompted philosophers to ascertain if any relation existed between magnetism and "the other forces of nature," as they were generally termed. We are consequently led to expect amongst the records of early experimental investigations some accounts which treat of the action of magnetisn on light.

\section{Early Experiments.}

When we seek for such accounts, however, we find that they are almost wholly absent from the literature of science, and this arises, I believe, from the great difficulty of the investigation and from the circumstance that only negative results were obtained, rather than that no such inquiry suggested itself or was undertaken. Even in quite recent times this inquiry has been prosecuted, but without success, by physicists who have published no account of their experiments. We may take it, therefore, that the inquiry is in itself an old one, although it is only now that it has been carried to a successful issue.

The earliest recorded attempt to solve this problem with which we are acquainted, is that of a celebrated British physicist whose name must for ever shed lustre on the annals of the Royal Institution-I speak of Michael Faraday. In order to understand the nature of the investigation which Faraday took in hand, and which has led up to the discourse of this evening, it is best to consider briefly some elementary facts concerning magnetism and light.

\section{Magnetic Field of Force.}

In the first place, I shall assume that we know in a general way what the peculiarities of a body are which lead us to say that it is magnetised, or a magnet. These are that, when freely suspended, it sets itself in a definite direction over the earth's surface, as illustrated by the compass needle, and that in the space around it there is "magnetic" force exerted on pieces of iron, and, in a smaller degree, on other substances. For this reason, we say that a magnet is surrounded by a magnetic field of force. The field of force is simply the space surrounding the magnet, and it extends to infinity in all directions

1 Friday evening diccourse delivered at the Royal Institution, May I2, by Thomas Preston, M.A., D.Sc., F.R.S. from the magnet. Near the magnet the force is strong, and far away from it the force is almost insensible ; and so we say that the field is strong at certain places near the magnet, and that it is weak at places far away from the magnet. The direction of the force at any point is the direction in which the north pole of another magnet would be urged if placed at that point, and the push which this pole experiences may be taken to represent the intensity or strength of the magnetic field at the point in question. This is represented diagrammatically by these drawings [referring to figures suspended before the audience], which show roughly the nature of the field of force surrounding an ordinary bar magnet, a horse-shoe magnet, and the much more powerful form-the electromagnet. It will be seen that the space outside the iron is filled with a system of curved lines running from the north pole to the south pole of the iron core. Where the lines are closest together there the magnetic force is strongest, and the direction of a line at any point is the direction of the resultant magnetic force at that pointthat is, the direction in which a north pole would be urged if placed at that point.

Faraday always pictured the magnetic field as filled with lines of force in this way, and the importance of the conception can scarcely be over-rated, for it leads us to view the magnetic action as being transmitted continuously through the intervention of some medium filling all space, rather than by the unintelligible process of direct action at a distance. This medium is called the ether; but as to what it is that is actually going on in the ether around a magnet, we cannot definitely say. It may be that there is a flow of ether along the lines of magnetic force, so that there is an outflow at one end of the magnet and an in-flow at the other, or it may be that the ether is spinning round the lines of force in the magnetic field. For our present purpose, it is not a matter of very much importance what the exact condition of the ether may be in a magnetic field, for if the ether in a magnetic field is either in some peculiar condition of strain or of motion, and if light consists of an undulatory motion propagated through this same ether, then it may be naturally expected that some action should take place when light is propagated through, or radiated in, a magnetic field of force. This is what Faraday suspected, and in order that we may appreciate the problem with which he had to deal, let us place ourselves in his position and ask ourselves the question: "In what manner can we test experimentally if there is any magnetic action on light?"

\section{Tests for Magnetic Action on Light.}

In answer to this question, the first thing that occurs to us is to pass a beam of ordinary light through the magnetic field, in some chosen directions, and examine by all the means at our disposal if any action has taken place. When this is done we find that no observable effect is produced. But the scientific investigator does not rest satisfied with one negative result. He varies the conditions of the experiment, and returns to the attack with renewed vigour and hopes. In our first trial we passed a beam of light through the air-filled space around the magnet, and we may vary this experiment either by removing the air altogether, and so causing the beam to traverse a vacuum, or we may replace the air by some dense transparent substance such as glass or water. Under these new conditions, we still fail to detect any influence of the magnetic field on a beam of ordinary light. This negative result might arise from the field of force being too weak to produce an observable effect, or it might be that the effect, if any effect really does exist, may be of such a character that it is impossible to detect it with ordinary light. In common light, the vibrations take place indifferently in all directions around the ray, and follow no law or order as to their type. They 